\title{
VIRTUAL KEYBOARD: AN INTERACTION METHOD USING
}

\section{CAMERA}

\author{
Shifa Panhalkar', Anjali Rathod², Akshay Garud ${ }^{3}$, Ritu Phadnis ${ }^{4}$ \\ ${ }^{1}$ Student, Computer, Anantrao Pawar College of Engineering, Maharastra, India \\ ${ }^{2}$ Student, Computer, Anantrao Pawar College of Engineering, Maharastra, India \\ ${ }^{3}$ Sponsored Guide, Computer, Opulent InfoTech, Maharastra, India \\ ${ }^{4}$ Internal Guide, Computer, Anantrao Pawar College of Engineering, Maharastra, India
}

\begin{abstract}
A virtual keyboard which doesn't require any external support and it can be operated by simply moving fingers on the paper on which keyboard is drawn, rather than typing on the physical keys of keyboard. In this paper, Camera is used to take input from the virtual Keyboard drawn on the paper. Here Camera is use to track the finger movements of typist to detect the users desired keystroke to type onto the Screen. Mapping of the keystroke is done to recognize the typed characters. The Proposed system is performed as: A Keyboard is drawn on a Plain Paper and user's finger is been covered using colored tape. HSV (Hue, Saturation and Value) values are been set for the colored tape used by the user using the scale provided to set value on the GUI panel. The finger covered using tape can be discernible from background using BLOB Analysis. As well as a grid is provided on the panel in the GUI to display the finger moment of the user. Then the user start typing on the paper keyboard and the typed character is been captured using camera. Then based the position of the finger placed in the grid the character is been recognized and then the recognized character is typed on the screen.
\end{abstract}

Keywords: Virtual Keyboard, Camera, Blob Analysis, HSV.

\section{INTRODUCTION}

The problem noticed in normal QWERTY keyboards is mainly due to its size, as they are mostly large in size and it really becomes difficult for a person to carry the keyboard from one place to the another Place. The chances of breaking the Keyboard also increase. The most important problematic situation for QWERTY system keyboard is basically its language restriction. The user has to install different language file based on the different language all the time so as to interact with the system using the language in which user wants to interact with the system. As invention of computers in recent years has been gone through a rapid miniaturization. Disks grew smaller in size, but only component of the computer is remained same for decades such as keyboard. As the miniaturization of a traditional keyboard is really very difficult so we have introduced virtual keyboard concept in this Paper.

A virtual keyboard doesn't have any physical support and which can be operated by the user via typing i.e. basically by just moving fingers on the paper. It is just an example of the latest computer trend of faster and smaller processing of keyboard. The basic idea behind the virtual keyboard is that the camera takes the input of finger movements of the typist to get the correct keystrokes. The keyboard is been projected on a flat surface and, as the then user touch the image of the key, the device detects the stroke and sends it [1].

\section{LITERATURE SURVEY}

There are many augmented virtual keyboard systems which have is been introduced earlier. Jun $\mathrm{Hu}$ et al.[1] has proposed a novel interactive projection system which enables bare-finger touch interaction on regular planar surfaces with one camera and one projector. Bare finger touch detection basically recovers the touch information just from 2-D image captured by camera. The Graphical user interface (GUI) buttons is been projected on the surface and is been distorted by the finger when clicked. M. Khalilbeigi et al. [2] has been introduced a rollable display; display size can be dynamically changed as per user desire. And it also investigates that how physical resizing of the display can be used as an input technique for interaction.

The hand shadow using ShadowPuppets [3] as input to mobile projector phones. $\mathrm{Z}$ Mo et al. [4] describes a technique which allows user to perform freehand drawing on a desk with gestures using SmartCanvas. Touch detection and hand detection [6] implemented to make any surface a touch sensitive display using camera and projector.

A depth touch is introduced by $\mathrm{H}$ Benko and A Wilson [5] which uses a depth-sensing camera. A flexible technique to calibrate the camera is described in [7]. Moving object can be detected [8] using BLOB in which single mode model based on BLOB analysis has been proposed from image sequences in complex environment. 


\section{METHODOLOGIES}

In this section, effective and simple technique are explained so to extract information captured by the camera

\subsection{BLOB Analysis}

BLOB analysis is based on analysis of image regions used for detecting regions in the digital image with different properties. The objects which are being inspected are clearly distinct from the background. Region can be defined as any subset of an image pixels and BLOB is a connected region. BLOB is been represented using the same region data type. BLOB analysis solution basically consists of three steps 1) Extraction: In Extraction Thresholding techniques are applied to obtain a region corresponding to objects which are to be inspected. 2) Refinement: then extracted region is enhanced using some of the region transformation techniques. 3) Analysis: the refined region is subjected to measurements and finally the results are computed. Main advantage of technique is its high flexibility and performance.

\subsection{HSV}

HSV is the most commonly used cylindrical-coordinate representation technique of points in RGB color model. These representation techniques rearrange the geometry of the RGB to be more relevant then the Cartesian representation. HSV are basically used in color pickers, Computer vision and image analysis. In this Paper we are using HSV as color picker to detect the color of the tap.

\section{PROPOSED SYSTEM}

The main objective of our project is to develop a desktop application for keyboard, which doesn't require any external hardware and it will be easy to use with reconfigurable options. The basic and the main focus of the paper is to know the problems related to the current virtual keyboard present the market and how we can solve these problems using different techniques. In this Paper we are presenting an idea in which Firstly a keyboard is been drawn on a plain Paper as shown in the Figure 1. Then the camera lens is been set facing on the drawn keyboard. And the camera and resolution is set from the GUI. Using "Start Camera" button the camera is been started.

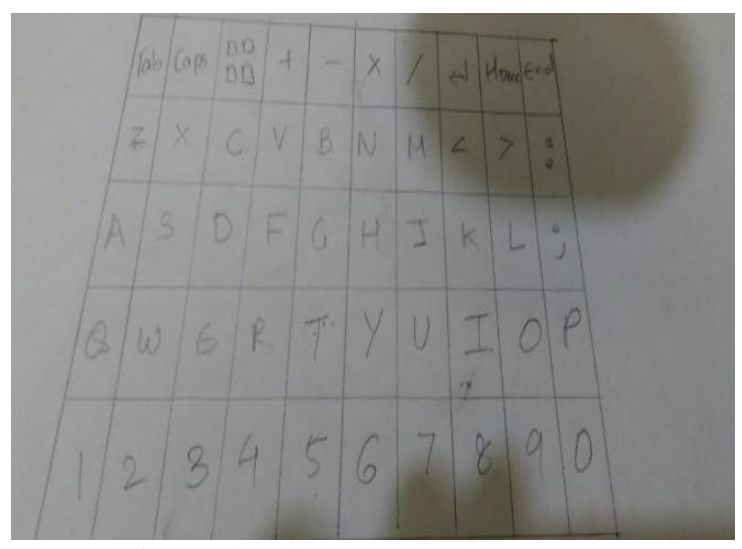

Figure 1: Keyboard drawn on paper

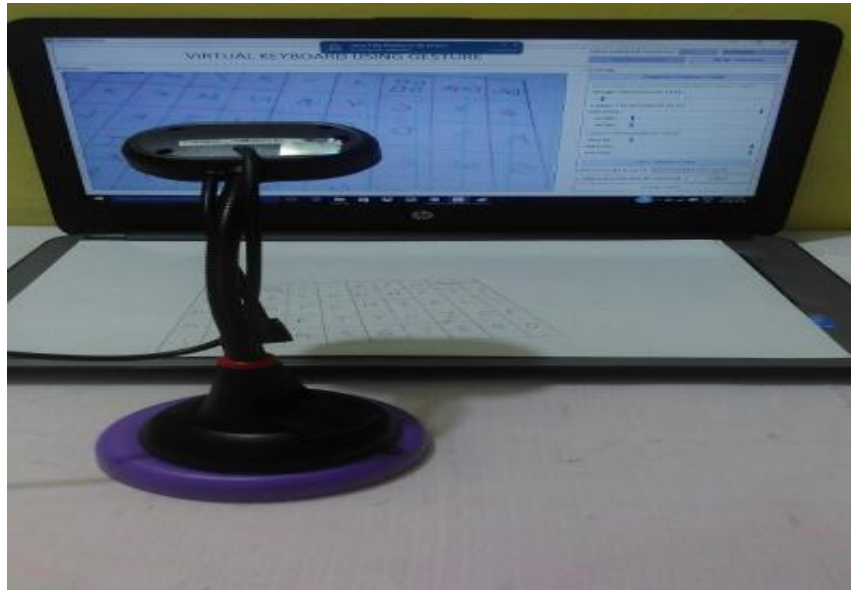

Figure 2: Setting the camera on keyboard.

Secondly, the users finger is been covered using the colored tape and HSV values are been set using the scale provided on the GUI screen. The GUI also consists of the panel with a grid on it which displays the keyboard drawn on the paper.

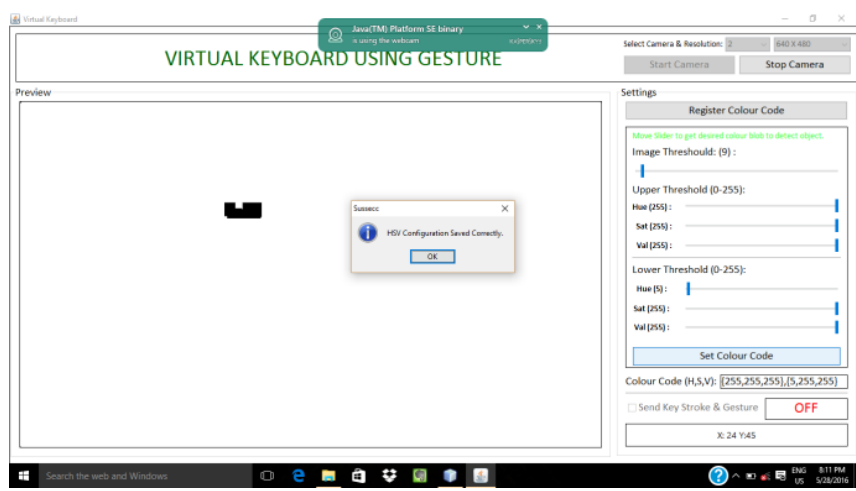

Figure 3: Setting the color code(HSV values)

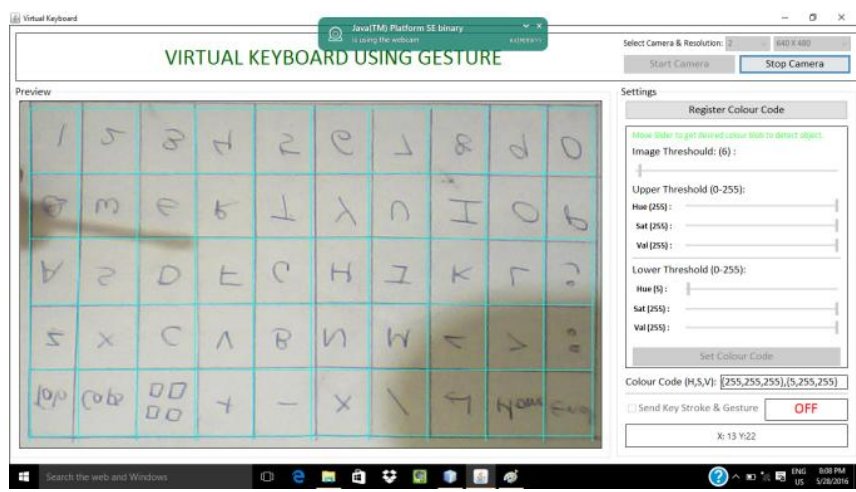

Figure 4: GUI Grid

User can start typing by moving the finger on the paper; the camera will take input as user moves its finger. Then the keystroke is being recognized based the users finger present in that particular grid. The keystroke is been matched using the "Robot" class. And the recognized letter are been typed on the Screen. 


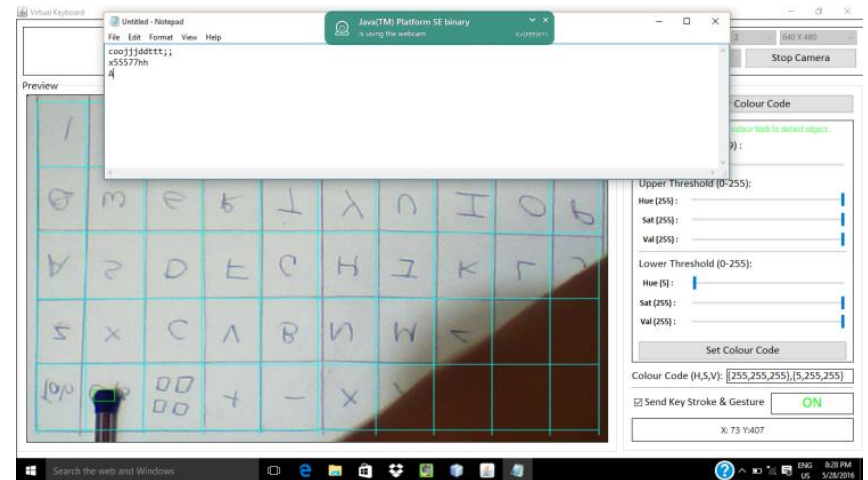

Figure 5: Typing onto Notepad

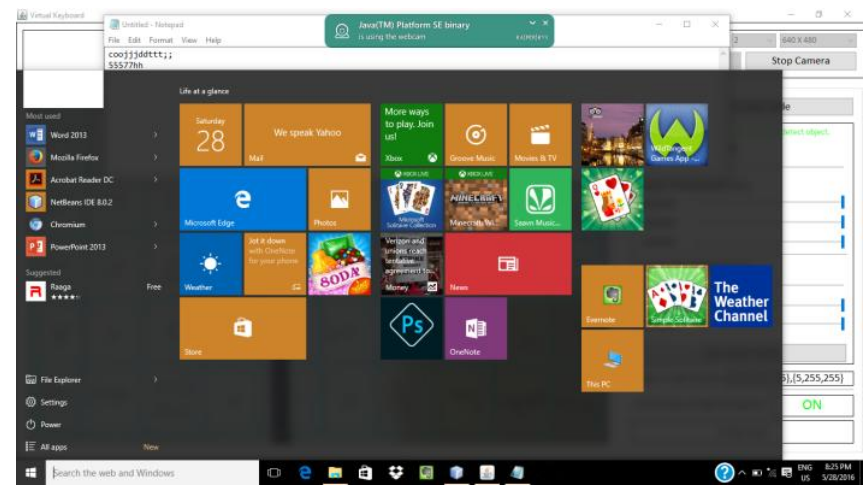

Figure 6: on selecting the windows key

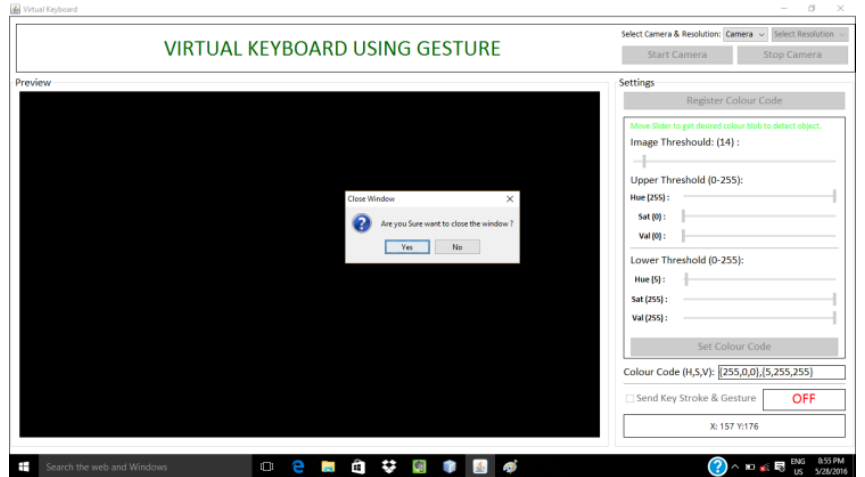

Figure 6: Closing the window

\section{CONCLUSIONS}

This paper basically illustrates the practical implementation of the virtual keyboard (VK).And this virtual keyboard demonstrates the upcoming tomorrow of human mobile devices as well as the human computer interaction in thi creation of virtual world. With the more increasing demand of small mobile devices, conventional data entry is required that are considerably flexible and easy to use without affecting.

The important properties like portability and mobility of the Keyboard Device. Virtual keyboard provide a convenience of compactness. As in this paper we are using a blank paper, so that user can draw the keyboard according to his or her comfort. Therefore we can say that these will make typing easier and almost a pleasure.

\section{ACKNOWLEDGEMENT}

Our thanks to Opulent InfoTech, college faculty members and friends for supporting this research

\section{REFERENCES}

[1]. Jun Hu, Guolin Li, Xiang Xie, Zhong Lv, and Zhihua Wang, "Bare-fingers Touch Detection by the Button's Distortion in a Projector-Camera System", in Proc. IEEE, vol. 24, No.4,2014.

[2]. M. Khalilbeigi, R. Lissermann, M. M“uhlh“auser, and J. Steimle, "Xpaaand: Interaction techniques for rollable displays," in Proc. ACM CHI, 2011, pp. 2729-2732.

[3]. L. G. Cowan and K. A. Li, "ShadowPuppets: Supporting collocated interaction with mobile projector phones using hand shadows," in Proc. ACM CHI, 2011, pp. 2707-2716.

[4].Z. Mo, J. P. Lewis, and U. Neumann, "SmartCanvas: A gesturedriven intelligent drawing desk system," in Proc. ACM IUI, 2005, pp. 239-243.

[5]. H. Benko and A. Wilson, "DepthTouch: Using depthsensing camera to enable freehand interactions on and above the interactive surface," in Proc. IEEE Workshop ITS, vol. 8, 2009.

[6] J. Dai and R. Chung, "Making any planer surface into a touch-sensitive display by a mere projector and camera," in Proc. IEEE Workshop CVPR, 2012, pp.35-42.

[7]Z. Zhang, "Flexible camera caliberation by viewing a plane from unknown orientations," in Proc. IEEE ICCV,1999, vol 1,PP. 666-673.

[8]R. I. Hartley, "In defence of the eight-point algorithm," IEEE Trans. Pattern Anal. Mach Intell, Vol 19, no. 6, pp.580-593, Jun 1997.

[9]T. Jia, N. SUN, M. CAO, "Moving object detection based on blob analysis," in Proc. IEEE ICAL, sept 2008.

[10] C. J. L. D. Alamo, L. J. F. Perez, L. A. R. Calla, W. R. R. Lovon, E. P. C. D. Computaction, U. N. D. S. Agustin, Arequipa, P. U. Salle, "A novel approach for image feature extraction using HSV model color and filters wavelets,"in Proc. IEEE, 2013

\section{BIOGRAPHIES}

Shifa Panhalkar, BE Computer Student at Anantrao Pawar College of Engineering, Pune, Maharashtra, India.

Anjali Rathod, BE Computer Student at Anantrao Pawar College of Engineering, Pune, Maharashtra, India.

Akshay Garud, Sponsored Guide, Opulent InfoTech, Pune, Maharashtra, India

Ritu Phadnis, Assistant Professor, at Anantrao Pawar College of Engineering, Pune, Maharashtra, India. 